\title{
Existence of Positive Solutions for Boundary Value Problem of Nonlinear Fractional $q$-Difference Equation ${ }^{*}$
}

\author{
Liu Yang \\ Department of Mathematics and Computing Sciences, Hengyang Normal University, Hengyang, China \\ Email: yangliu19731974@yahoo.com.cn
}

Received April 14, 2013; revised May 14, 2013; accetped May 21, 2013

Copyright (c) 2013 Liu Yang. This is an open access article distributed under the Creative Commons Attribution License, which permits unrestricted use, distribution, and reproduction in any medium, provided the original work is properly cited.

\begin{abstract}
In this paper, we investigate the existence of positive solutions for a class of nonlinear $q$-fractional boundary value problem. By using some fixed point theorems on cone, some existence results of positive solutions are obtained.
\end{abstract}

Keywords: Fractional q-Difference Equation; Positive Solution; Fixed Point Theorems on Cone

\section{Introduction}

Considering the following boundary value problem of nonlinear fractional $q$-difference equation:

$$
\left\{\begin{array}{l}
\left(D_{q}^{\alpha} y\right)(x)=-f(x, y(x)), \quad 0<x<1,3<\alpha \leq 4, \\
y(0)=\left(D_{q} y\right)(0)=\left(D_{q}^{2} y\right)(0)=0,\left(D_{q}^{3} y\right)(1)=0,
\end{array}\right.
$$

where $f$ is a nonnegative continuous function and $D_{q}^{\alpha}$ is the fractional $q$-derivative of the Riemann-Liouville type.

Fractional differential calculus is a discipline to which many researchers are dedicating their time, perhaps because of its demonstrated applications in various fields of science and engineering [1]. Recently, there are many papers dealing with the boundary value problem of fractional differential equations, see [2-5] and references therein.

The $q$-difference calculus or quantum calculus is an old subject that was initially developed by Jackson [6,7], and basic definitions and properties of $q$-difference calculus can be found in [8]. The fractional $q$-difference calculus had its origin in the works by Al-Salam [9] and Agarwal [10]. More recently, maybe due to the explosion in research within the fractional differential calculus setting, new developments in this theory of fractional $q$ difference calculus were made, see $[11,12]$.

The question of the existence of positive solutions for

"This work was supported by the Natural Science Foundation of Hunan Province (12JJ9001), Hunan Provincial Science and Technology Department of Science and Technology Project (2012SK3117) and Construct program of the key discipline in Hunan Province. fractional q-difference boundary value problems is in its infancy, see [13-16]. No contributions exist, as far as we know, concerning the existence of positive solutions for problem (P).

This paper is organized as follows. In Section 2, some preliminaries are presented. In Section 3, we discuss the existence of positive solutions for problem (P).

\section{Preliminaries}

Let $q \in(0,1)$ and define

$$
[a]_{q}=\frac{1-q^{a}}{1-q}, \quad a \in \mathbb{R} .
$$

The $q$-analogue of the power function $(a-b)^{n}$ with $n \in \mathbb{N}_{0}$ is

$$
(a-b)^{0}=1, \quad(a-b)^{n}=\prod_{k=0}^{n-1}\left(a-b q^{k}\right), \quad n \in \mathbb{N}, a, b \in \mathbb{R} .
$$

More generally, if $a \in \mathbb{R}$, then

$$
(a-b)^{(\alpha)}=a^{\alpha} \prod_{n=0}^{\infty} \frac{a-b q^{n}}{a-b q^{\alpha+n}} .
$$

Note that, if $b=0$ then $a^{(\alpha)}=a^{\alpha}$. The $q$-gamma function is defined by

$$
\Gamma_{q}(x)=\frac{(1-q)^{(x-1)}}{(1-q)^{x-1}}, \quad x \in \mathbb{R} \backslash\{0,-1,-2, \cdots\}
$$

and satisfies $\Gamma_{q}(x+1)=[x]_{q} \Gamma_{q}(x)$. The $q$-derivative of a function $f$ is here defined by 


$$
\left(D_{q} f\right)(x)=\frac{f(x)-f(q x)}{(1-q) x}, \quad\left(D_{q} f\right)(0)=\lim _{x \rightarrow 0}\left(D_{q} f\right)(x)
$$

and $q$-derivative of higher order by

$$
\left(D_{q}^{0} f\right)(x)=f(x), \quad\left(D_{q}^{n} f\right)(x)=D_{q}\left(D_{q}^{n-1} f\right)(x), n \in \mathbb{N} .
$$

The $q$-integral of a function $f$ defined in the interval $[0, b]$ is given by

$$
\left(I_{q} f\right)(x)=\int_{0}^{x} f(t) d_{q} t=x(1-q) \sum_{n=0}^{\infty} f\left(x q^{n}\right) q^{n}, x \in[0, b] .
$$

If $a \in[0, b]$ and $f$ defined in the interval $[0, b]$, its integral from $a$ to $b$ is defined by

$$
\int_{a}^{b} f(t) d_{q} t=\int_{0}^{b} f(t) d_{q} t-\int_{0}^{a} f(t) d_{q} t .
$$

Remark 2.1. (see [17]) If $a=b q^{n}, n \in \mathbb{N}$ and $f(t) \geq g(t)$ on $[a, b]$, then

$$
\int_{a}^{b} f(t) d_{q} t \geq \int_{a}^{b} g(t) d_{q} t .
$$

Similarly as done for derivatives, an operator $I_{q}^{n}$ can be defined, namely,

$$
\left(I_{q}^{0} f\right)(x)=f(x), \quad\left(I_{q}^{n} f\right)(x)=I_{q}\left(I_{q}^{n-1} f\right)(x), n \in \mathbb{N} .
$$

The fundamental theorem of calculus applies to these operators $I_{q}$ and $D_{q}$, i.e.,

$$
\left(D_{q} I_{q} f\right)(x)=f(x)
$$

and if $f$ is continuous at $x=0$, then

$$
\left(I_{q} D_{q} f\right)(x)=f(x)-f(0) .
$$

Basic properties of the two operators can be found in [14]. We now point out three formulas that will be used later $\left({ }_{i} D_{q}\right.$ denotes the derivative with respect to variable i)

$$
\begin{gathered}
{[a(t-s)]^{\alpha}=a^{\alpha}(t-s)^{(\alpha)},} \\
{ }_{t} D_{q}(t-s)^{(\alpha)}=[\alpha]_{q}(t-s)^{(\alpha-1)}, \\
\left(-x D_{q} \int_{0}^{x} f(x, t) d_{q} t\right)(x)=\int_{0}^{x}-x D_{q} f(x, t) d_{q} t+f(q x, x) .
\end{gathered}
$$

Remark 2.2. (see [14]) We note that if $\alpha>0$ and $a \leq b \leq t$, then

$$
(t-a)^{(\alpha)} \geq(t-b)^{(\alpha)}
$$

Definition 2.3. (see [10]) Let $\alpha \geq 0$ and $f$ be a function defined on $[0,1]$. The fractional $q$-integral of the Riemann-Liouville type is $\left(I_{q}^{0} f\right)(x)=f(x)$ and

$$
\begin{aligned}
& \left(I_{q}^{\alpha} f\right)(x)=\frac{1}{\Gamma_{q}(\alpha)} \int_{0}^{x}(x-q t)^{(\alpha-1)} f(t) \mathrm{d}_{q} t, \\
& \alpha>0, x \in[0,1] .
\end{aligned}
$$

Definition 2.4. (see [14-16]) The fractional $q$-derivative of the Riemann-Liouville type of order $\alpha \geq 0$ is defined by $\left(D_{q}^{0} f\right)(x)=f(x)$ and

$$
\left(D_{q}^{\alpha} f\right)(x)=\left(D_{q}^{m} I_{q}^{m-\alpha} f\right)(x), \quad \alpha>0,
$$

where $m$ is the smallest integer greater than or equal to $\alpha$.

Next, we list some properties that are already known in the literature.

Lemma 2.5. (see [14-16]) Let $\alpha, \beta \geq 0$ and $f$ be a function defined on $[0,1]$, Then, the next formulas hold:

1) $\left(I_{q}^{\beta} I_{q}^{\alpha} f\right)(x)=\left(I_{q}^{\alpha+\beta} f\right)(x)$,

2) $\left(D_{q}^{\alpha} I_{q}^{\alpha} f\right)(x)=f(x)$.

Lemma 2.6. (see [14-16]) Let $\alpha>0$ and $p$ be a positive integer. Then, the following equality holds:

$$
\begin{aligned}
& \left(I_{q}^{\alpha} D_{q}^{p} f\right)(x) \\
= & \left(D_{q}^{p} I_{q}^{\alpha} f\right)(x)-\sum_{k=0}^{p-1} \frac{x^{\alpha-p+k}}{\Gamma_{q}(\alpha+k-p+1)}\left(D_{q}^{k} f\right)(0) .
\end{aligned}
$$

Let $p=4$, in view of Lemma 2.5 and Lemma 2.6, we see that

$$
\begin{aligned}
& \left(D_{q}^{\alpha} y\right)(x)=-f(x, y(x)) \\
& \Leftrightarrow\left(I_{q}^{\alpha} D_{q}^{4} I_{q}^{4-\alpha} y\right)(x)=-I_{q}^{\alpha} f(x, y(x)) \\
& \Leftrightarrow y(x)=c_{1} x^{\alpha-1}+c_{2} x^{\alpha-2}+c_{3} x^{\alpha-3} \\
& \quad+c_{4} x^{\alpha-4}-\frac{1}{\Gamma_{q}(\alpha)} \int_{0}^{x}(x-q t)^{(\alpha-1)} f(t, y(t)) \mathrm{d}_{q} t
\end{aligned}
$$

for some constants $c_{1}, c_{2}, c_{3}, c_{4} \in \mathbb{R}$. Using the boundary condition $y(0)=0$ we have $c_{4}=0$. Differentiating both side of the above equality, one gets

$$
\begin{aligned}
D_{q} y= & {[\alpha-1]_{q} c_{1} x^{\alpha-2}+[\alpha-2]_{q} c_{2} x^{\alpha-3}+[\alpha-3]_{q} c_{3} x^{\alpha-4} } \\
& -\frac{1}{\Gamma_{q}(\alpha)} \int_{0}^{x}[\alpha-1]_{q}(x-q t)^{(\alpha-2)} f(t, y(t)) \mathrm{d}_{q} t .
\end{aligned}
$$

Using the boundary condition $\left(D_{q} y\right)(0)=0$, we have $c_{3}=0$. similarly, we have $c_{2}=0$. From

$$
\begin{aligned}
\left(D_{q}^{3}\right) y= & {[\alpha-1]_{q}[\alpha-2]_{q}[\alpha-3]_{q} c_{1} x^{\alpha-4} } \\
& -\frac{1}{\Gamma_{q}(\alpha)} \int_{0}^{x}[\alpha-1]_{q}[\alpha-2]_{q}[\alpha-3]_{q} \\
& \cdot(x-q t)^{(\alpha-4)} f(t, y(t)) \mathrm{d}_{q} t
\end{aligned}
$$


and boundary value problem $\left(D_{q}^{3}\right) y(1)=0$, one can obtain

$$
c_{1}=\frac{1}{\Gamma_{q}(\alpha)} \int_{0}^{1}(1-q t)^{(\alpha-4)} f(t, y(t)) \mathrm{d}_{q} t .
$$

Putting all things together we finally have

$$
\begin{aligned}
& y x=\left(\frac{1}{\Gamma_{q}(\alpha)} \int_{0}^{1}(1-q t)^{(\alpha-4)} f(t, y(t)) d_{q} t\right) x^{\alpha-1} \\
& -\frac{1}{\Gamma_{q}(\alpha)} \int_{0}^{x}(x-q t)^{(\alpha-1)} f(t, y(t)) d_{q} t \\
& =\frac{1}{\Gamma_{q}(\alpha)}\left[\int_{0}^{x}\left((1-q t)^{(\alpha-4)} x^{\alpha-1}-(x-q t)^{(\alpha-1)}\right) f(t, y(t)) d_{q} t\right] \\
& +\int_{x}^{1}(1-q t)^{(\alpha-4)} x^{\alpha-1} f(t, y(t)) \mathrm{d}_{q} t .
\end{aligned}
$$

If we define a function $G$ by

$$
G(x, t)=\frac{1}{\Gamma_{q}(\alpha)}\left\{\begin{array}{l}
(1-t)^{(\alpha-4)} x^{\alpha-1}-(x-t)^{(\alpha-1)}, \\
0 \leq t \leq x \leq 1, \\
(1-t)^{(\alpha-4)} x^{\alpha-1}, \\
0 \leq x \leq t \leq 1 .
\end{array}\right.
$$

Hence, in order to solve the problem (P), it is sufficient to find positive solutions of the following integral equation

$$
y(t)=\int_{0}^{1} G(x, q t) f(t, y(t)) \mathrm{d}_{q} t .
$$

Some properties of the function $G$ needed in the sequel are now stated and proved.

Lemma 2.7. Function $G$ defined above satisfies the following conditions:

$$
\begin{gathered}
G(x, q t) \geq 0, \quad G(x, q t) \leq G(1, q t), \\
\text { for all } 0 \leq x, t \leq 1, \\
G(x, q t) \geq x^{\alpha-1} G(1, q t) \text {, for all } 0 \leq x, t \leq 1 .
\end{gathered}
$$

Proof. Let

$$
g_{1}(x, t)=(1-t)^{(\alpha-4)} x^{\alpha-1}-(x-t)^{(\alpha-1)}, 0 \leq t \leq x \leq 1(2.2
$$

and

$$
g_{2}(x, t)=(1-t)^{(\alpha-4)} x^{\alpha-1}, 0 \leq x \leq t \leq 1 .
$$

It is clear that $g_{2}(x, q t) \geq 0$. Now, $g_{2}(0, q t)=0$. For $x \neq 0$, in view of (2.3) and Remark 2.2, we have

$$
\begin{aligned}
g_{1}(x, q t) & =(1-q t)^{(\alpha-4)} x^{\alpha-1}-x^{\alpha-1}\left(x-\frac{q t}{x}\right)^{(\alpha-1)} \\
& \geq x^{\alpha-1}\left[(1-q t)^{(\alpha-4)}-(1-q t)^{(\alpha-1)}\right] \geq 0 .
\end{aligned}
$$

Therefore, $G(x, t) \geq 0$. Moreover,

$$
\begin{aligned}
& { }_{x} D_{q} g_{1}(x, q t) \\
= & (1-q t)^{(\alpha-4)}[\alpha-1]_{q} x^{\alpha-2}-[\alpha-1]_{q} x^{\alpha-2}\left(x-\frac{q t}{x}\right)^{(\alpha-2)} \\
\geq & {[\alpha-1]_{q} x^{\alpha-2}\left[(1-q t)^{(\alpha-4)}-(1-q t)^{(\alpha-2)}\right] \geq 0 . }
\end{aligned}
$$

i.e., $g_{1}(x, q t)$ is an increasing function of $x$. Obviously, $g_{2}(x, q t)$ is increasing in $x$, therefore, $G(x, q t)$ is an increasing function of $x$ for fixed $t \in[0,1]$. This concludes the proof of (2.22).

Suppose now that $x \geq q t$, then

$$
\begin{aligned}
\frac{G(x, q t)}{G(1, q t)}= & \frac{(1-q t)^{(\alpha-4)} x^{\alpha-1}-(x-q t)^{(\alpha-1)}}{(1-q t)^{(\alpha-4)}-(1-q t)^{(\alpha-1)}} \\
= & \frac{x^{\alpha-1}\left[(1-q t)^{(\alpha-4)}-\left(1-\frac{q t}{x}\right)^{(\alpha-1)}\right]}{(1-q t)^{(\alpha-4)}-(1-q t)^{(\alpha-1)}} \\
& \geq \frac{x^{\alpha-1}\left[(1-q t)^{(\alpha-4)}-(1-q t)^{(\alpha-1)}\right]}{(1-q t)^{(\alpha-4)}-(1-q t)^{(\alpha-1)}}=x^{\alpha-1} .
\end{aligned}
$$

If $x \leq q t$, then

$$
\frac{G(x, q t)}{G(1, q t)}=x^{\alpha-1}
$$

and this finishes the proof of (2.23).

Let $\mathfrak{I}=C[0,1]$ be the Banach space endowed with norm $\|u\|=\sup _{t \in[0,1]}|u(t)|$. Define the cone $C$ by

$$
C=\left\{u \in \mathfrak{J}: u(x) \geq x^{\alpha-1}\|u\|\right\} .
$$

It follows from the non-negativeness and continuity of $G$ and $f$ that the operator $T: C \rightarrow \mathfrak{I}$ defined by

$$
(T u)(x)=\int_{0}^{1} G(x, q t) f(t, u(t)) \mathrm{d}_{q} t
$$

is completely continuous [18]. Moreover, for $u \in C$, in view of (2.22) and (2.23), we have $(T u)(x) \geq 0$ on $[0,1]$ and

$$
\begin{aligned}
(T u)(x) & =\int_{0}^{1} G(x, q t) f(t, u(t)) d_{q} t \\
& \geq x^{\alpha-1} \int_{0}^{1} G(1, q t) f(t, u(t)) d_{q} t \\
& \geq x^{\alpha-1}\|T u\|,
\end{aligned}
$$

that is $T(C) \subset C$.

Lemma 2.8. (see [19]) Let $E$ be a Banach space, $P \subset E$ a cone, and $\Omega_{1}, \Omega_{2}$ two bounded open balls of $E$ centered at the origin with $\bar{\Omega}_{1} \subset \Omega_{2}$. Suppose that $T: P \cap\left(\bar{\Omega}_{2} \backslash \Omega_{1}\right) \rightarrow P$ is a completely continuous op- 
erator such that either

1) $\|T u\| \leq\|u\|, u \in P \bigcap \partial \Omega_{1}$ and $\|T u\| \geq\|u\|, u \in P \bigcap \partial \Omega_{2}$, or

2) $\|T u\| \geq\|u\|, u \in P \bigcap \partial \Omega_{1}$ and $\|T u\| \leq\|u\|, u \in P \bigcap \partial \Omega_{2}$ holds. Then $T$ has a fixed point in $P \cap\left(\bar{\Omega}_{2} \backslash \Omega_{1}\right)$.

\section{Main Results}

Let $\Omega_{i}=\left\{y \in C[0,1]:\|y\| \leq r_{1}\right\}, i=1,2,3,4$, where $r_{i}>0$ will be defined later.

Theorem 3.1. Suppose that $f(t, u)$ is a nonnegative continuous function on $[0,1] \times[0, \infty)$. In addition, suppose that one of the following two conditions holds:

(H1)

$$
\begin{aligned}
& \lim _{y \rightarrow 0} \min _{x \in[0,1]} \frac{f(x, y)}{y}=\infty, \\
& \lim _{y \rightarrow \infty} \min _{x \in[0,1]} \frac{f(x, y)}{y}=0 ; \\
& \lim _{y \rightarrow 0} \min _{x \in[0,1]} \frac{f(x, y)}{y}=0, \\
& \lim _{y \rightarrow \infty} \min _{x \in[0,1]} \frac{f(x, y)}{y}=\infty .
\end{aligned}
$$

Then problem $(\mathrm{P})$ has at least one positive solution.

Proof. Note that the operator $T(C) \subset C$ is completely continuous. Now, assume that condition (H1) holds. Since $\lim _{y \rightarrow 0} \min _{x \in[0,1]} \frac{f(x, y)}{y}=\infty$, there exists an $r_{1}>0$ such that

$$
f(x, y) \geq \varepsilon_{1} y, \text { for } x \in[0,1], 0 \leq y \leq r_{1},
$$

where the constant $\varepsilon_{1}>0$ such that

$$
\varepsilon_{1} \int_{0}^{1} G(1, q t) t^{\alpha-1} d_{q} t>1 .
$$

Thus,

$$
f(x, y(x)) \geq \varepsilon_{1} y, \text { for } y \in C \cap \partial \Omega_{1}, x \in[0,1] .
$$

This, together with the definitions of $C$ and Lemma 2.7, implies that for any $y \in C \cap \partial \Omega_{1}$,

$$
\begin{aligned}
\|T y\| & =\max _{x \in[0,1]} \int_{0}^{1} G(x, q t) f(t, y(t)) d_{q} t \\
& =\int_{0}^{1} G(1, q t) f(t, y(t)) d_{q} t \\
& \geq \varepsilon_{1} \int_{0}^{1} G(1, q t) y(t) d_{q} t \\
& \geq \varepsilon_{1} \int_{0}^{1} G(1, q t) t^{\alpha-1} d_{q} t\|y\| \\
& \geq\|y\| .
\end{aligned}
$$

That is, for any $y \in C \cap \partial \Omega_{1}, \quad\|T y\| \geq\|y\|$.

On the other hand, from $\lim _{y \rightarrow \infty} \max _{x \in[0,1]} \frac{f(x, y)}{y}=0$, it follows that there exists a $L_{1}>0$ such that

$$
f(x, y) \leq \varepsilon_{2} y \text { for } x \in[0,1], y \geq L_{1},
$$

where the constant $\varepsilon_{2}>0$ satisfies

$$
\varepsilon_{2} \int_{0}^{1} G(1, q t) d_{q} t<\frac{1}{2} .
$$

Let $L_{2}=\max _{x \in[0,1], y \in\left[0, L_{1}\right]}\{f(x, y)\}$. Then we have

$$
f(x, y) \leq \varepsilon_{2} y+L_{2} \text {, for } x \in[0,1], y \geq 0 .
$$

Let $r_{2}=\max \left\{2 r_{1}, 2 L_{2} \int_{0}^{1} G(1, q t) d_{q} t\right\}$ and $y \in C \cap \partial \Omega_{2}$, then

$$
\begin{aligned}
& \|T y\|=\max _{x \in[0,1]} \int_{0}^{1} G(x, q t) f(t, y(t)) d_{q} t \\
& =\int_{0}^{1} G(1, q t) f(t, y(t)) d_{q} t \leq \int_{0}^{1} G(1, q t)\left(\varepsilon_{2} y+L_{2}\right) d_{q} t \\
& \leq \varepsilon_{2} \int_{0}^{1} G(1, q t) d_{q} t\|y\|+L_{2} \int_{0}^{1} G(1, q t) d_{q} t \leq\|y\| .
\end{aligned}
$$

Thus, the operator $T$ satisfies condition of Lemma 2.8. Consequently, the operator $T$ has at least one fixed point $y \in C \cap\left((\bar{\Omega}) \backslash \Omega_{1}\right)$, which is one positive solution of the problem $(\mathrm{P})$.

Next, we suppose that condition (H2) holds. The proof is similar to that of the case in which (H1) holds and will only be sketched here. Let $\tau=q^{n}$ with $n \in \mathbb{N}$. Select two positive constants $\varepsilon_{3}, \varepsilon_{4}$ with $\varepsilon_{3} \int_{0}^{1} G(1, q t) d_{q} t<1$ and $\varepsilon_{4} \tau^{\alpha-1} \int_{\tau}^{1} G(1, q t) d_{q} t>1$, respectively. Then, there exist two positive constants $r_{3}$ and $L_{3}$ such that

$$
\begin{gathered}
f(x, y) \leq \varepsilon_{3} y, \text { for } x \in[0,1], 0 \leq y \leq r_{3}, \\
f(x, y) \geq \varepsilon_{4} y, \text { for } x \in[0,1], y \geq L_{3} .
\end{gathered}
$$

It follows from that for $y \in C \cap \partial \Omega_{3}$,

$$
\begin{aligned}
& \|T y\| \\
= & \int_{0}^{1} G(1, q t) f(t, y(t)) d_{q} t \leq \varepsilon_{3} \int_{0}^{1} G(1, q t) y(t) d_{q} t \leq\|y\| .
\end{aligned}
$$

In addition, let $r_{4}=\max \left\{2 r_{3}, L_{3} \tau^{1-\alpha}\right\}$. If $y \in C \bigcap \partial \Omega_{r_{4}}$, then

$$
y(x) \geq \tau^{\alpha-1} r_{4} \geq L_{3}, \text { for } x \in[\tau, 1]
$$

and

$$
f(x, y(x)) \geq \varepsilon_{4} y, \text { for } x \in[\tau, 1], y \in C \cap \partial \Omega_{4} .
$$

In view of Remark 2.1, for $y \in C \cap \partial \Omega_{4}$, we have

$$
\begin{aligned}
& \|T y\|=\max _{x \in[0,1]} \int_{0}^{1} G(x, q t) f(t, y(t)) d_{q} t \\
& =\int_{0}^{1} G(1, q t) f(t, y(t)) d_{q} t \geq \varepsilon_{4} \int_{\tau}^{1} G(1, q t) y(t) d_{q} t \\
& \geq \varepsilon_{4} \int_{\tau}^{1} G(1, q t) t^{\alpha-1} d_{q} t\|y\| \geq \varepsilon_{4} \tau^{\alpha-1} \int_{\tau}^{1} G(1, q t) d_{q} t\|y\| \\
& \geq\|y\| .
\end{aligned}
$$


Thus, $\|T y\| \geq\|y\|$ for $y \in C \cap \partial \Omega_{4}$. Consequently, the operator $T$ has at least one fixed point $y \in C \cap(\bar{\Omega})_{4} \backslash \Omega_{3}$, which is one positive solution of the problem (P).

\section{Example 3.2}

$$
\left\{\begin{array}{l}
\left(D_{0.5}^{3.5} y\right)(x)=-f(x, y(x)), \quad 0<x<1, \\
y(0)=\left(D_{0.5} y\right)(0)=\left(D_{0.5}^{2} y\right)(0)=0,\left(D_{0.5}^{3} y\right)(1)=0,
\end{array}\right.
$$

where $f(x, y(x))=y^{\frac{1}{2}}(x)+\log (1+y(x))$. Obviously,

$$
\begin{aligned}
& \lim _{y \rightarrow 0} \max _{x \in[0,1]} \frac{f(x, y)}{y}=\infty, \\
& \lim _{y \rightarrow \infty} \max _{x \in[0,1]} \frac{f(x, y)}{y}=0 .
\end{aligned}
$$

Thus, by the first part of Theorem 3.1, we can get that the problem above has at least one positive solution.

\section{REFERENCES}

[1] I. Podlubny, "Fractional Differential Equations," Academic Press, San Diego, 1999.

[2] M. El-Shahed, "Existence of Solution for a Boundary Value Problem of Fractional Order," Advances in Applied Mathematical Analysis, Vol. 2, No. 1, 2007, pp. 1-8.

[3] S. Zhang, "Existence of Solution for a Boundary Value Problem of Fractional Order," Acta Mathematica Scientia, Vol. 26, No. 2, 2006, pp. 220-228. http://dx.doi.org/10.1016/S0252-9602(06)60044-1

[4] M. El-Shahed and F. M. Al-Askar, "On the Existence of Positive Solutions for a Boundary Value Problem of Fracntional Order," International Journal of Mathematical Analysis, Vol. 4, No. 13-16, 2010, pp. 671-678.

[5] Z. B. Bai and H. S. Lü, "Positive Solutions for Boundary Value Problem of Nonlinear Fractional Differential Equation," Journal of Mathematical Analysis and Applications, Vol. 311, No. 2, 2005, pp. 495-505.

http://dx.doi.org/10.1016/j.jmaa.2005.02.052

[6] F. H. Jackson, "On $q$-Functions and a Certain Difference Operator,” Transactions of the Royal Society Edinburgh, Vol. 46, No. 2, 1909, pp. 253-281. http://dx.doi.org/10.1017/S0080456800002751

[7] R. Jackson, “On $q$-Definite Integrals,” Quarterly Journal of Pure and Applied Mathematics, Vol. 41, 1910, pp.
193-203.

[8] V. Kac and P. Cheung, "Quantum Calculus,” Springer, New York, 2002.

http://dx.doi.org/10.1007/978-1-4613-0071-7

[9] W. A. Al-Salam, "Some Fractional $q$-Integrals and $q$ Derivatives," Proceedings of the Edinburgh Mathematical Society, Vol. 15, No. 2, 1967, pp. 135-140. http://dx.doi.org/10.1017/S0013091500011469

[10] R. P. Agarwal, "Certain Fractional $q$-Integrals and $q$ Derivatives," Mathematical Proceedings of the Cambridge Philosophical Society, Vol. 66, No. 2, 1969, pp. 365-370. http://dx.doi.org/10.1017/S0305004100045060

[11] P. M. Rajkovi, S. D. Marinkovi and M. S. Stankovi, “On q-Analogues of Caputo Derivative and Mittag-Leffler Function,” Fractional Calculus Applied Analysis, Vol. 10, No. 4, 2007, pp. 359-373.

[12] F. M. Atici and P. W. Eloe, "Fractional $q$-Calculus on a Time Scale,” Journal of Nonlinear Mathematical Physics, Vol. 14, No. 3, 2007, pp. 333-370.

[13] M. El-Shahed and H. A. Hassan, "Positive Solutions of q-Difference Equation," Proceedings of the American Mathematical Society, Vol. 138, No. 5, 2010, pp. 17331738. http://dx.doi.org/10.1090/S0002-9939-09-10185-5

[14] R. A. C. Ferreira, "Nontrivial Solutions for Fractional $q$ Difference Boundary Value Problems," Electronic Journal of Qualitative Theory of Differential Equations, No. 70, 2010, pp. 1-10.

[15] R. A. C. Ferreira, "Positive Solutions for a Class of Boundary Value Problems with Fractional q-Differences," Computers and Mathematics with Applications, Vol. 61, No. 2, 2011, pp. 367-373.

http://dx.doi.org/10.1016/j.camwa.2010.11.012

[16] M. El-Shahed and F. M. Al-Askar, "Positive Solutions for Boundary Value Problem of Nonlinear Fractional $q$-Differences,” ISRN Mathematical Analysis, Vol. 2011, 2011, pp. 1-12. http://dx.doi.org/10.5402/2011/385459

[17] H. Gauchman, "Integral Inequality in q-Calculus," Computers \& Mathematics with Applications, Vol. 47, No. 2-3, 2004, pp. 281-300. http://dx.doi.org/10.1016/S0898-1221(04)90025-9

[18] M. D. Rus, "A Note on the Existence of Positive Solutions of Fredholm Integral Equations," Fixed Point Theory, Vol. 5, No. 2, 2004, pp. 369-377.

[19] D. J. Guo and V. Lakshmikantham, "Nonlinear Problems in abstrAct Cones,” Academic Press, San Diego, 1988. 